\title{
Flexible job-shop scheduling with learning and forgetting effect by Multi-Agent System
}

\author{
Paolo Renna ${ }^{a^{*}}$
}

${ }^{a}$ School of Engineering, University of Basilicata, 85100 - Potenza, Italy

\begin{tabular}{l}
\hline C H R O N I C L E \\
\hline Article history: \\
Received March 22019 \\
Received in Revised Format \\
March 132019 \\
Accepted March 292019 \\
Available online \\
March 29 2019 \\
\hline Keywords: \\
Flexible job-shop \\
Scheduling \\
Learning \\
Forgetting \\
Multi Agent System \\
Simulation \\
\hline
\end{tabular}
\begin{abstract}
A B S T R A C T
The processing time of the machine is assumed fixed in several studies. In many real industrial applications, the processing time is affected by learning and forgetting effects. This research proposes a scheduling approach to support a manufacturing system under learning/forgetting effect. The approach is supported by a Multi-Agent System to perform the scheduling activities in a quasi-real-time and in general manufacturing systems. A simulation environment is developed to test the proposed approach and the results are compared with a benchmark model for evaluating several performance measures of the manufacturing system. The simulation results highlight how the proposed approach improves all the performance measures under different conditions of inter-arrival time, learning and forgetting rates. A complete Analysis of the Variance highlights the main effects on the performance measures to support the decision maker of the manufacturing system.
\end{abstract}

\section{Introduction}

Manufacturing processes with human contributions can be affected by the impact of learning and forgetting effects on the performance. The importance of the worker learning effects have been observed in several industrial contexts (Yelle, 1979). Globerson et al. (1989) proposed one of the first studies on the forgetting effect by considering the impact of the production systems which depends on the break length and the level of experience gained prior to the break. These effects are common in labor-intensive production assembly systems. The learning and forgetting rates depend on the characteristics of the individual worker and the specific task affected by the learning and forgetting effect. In industrial systems, this means that the learning and forgetting rates can be more heterogeneous on the job shop. The impact of the different learning and forgetting rates of the workers on the production systems are studied by Shafer et al. (2001) and Nembhard and Shafer (2008). Their works used simulation models to consider the different rates of the workers instead of assuming the average rate for the entire workforce and over different tasks of the production system. A comprehensive survey on the learning model proposed in the literature to model learning effects as realistic as possible was provided in Biskup (2008). This survey discussed the scheduling problem for the different learning models and the solutions based

* Corresponding author Tel. : +390971205143

E-mail: paolo.renna@unibas.it (P. Renna)

2019 Growing Science Ltd.

doi: $10.5267 /$ j.ijiec.2019.3.003 
mainly on dispatching rules. The scheduling approaches have the ability to operate in a volatile business environment with higher competition, more differentiation and shorter product life cycle (KPMGInt., Amstelveen, the Netherlands, 2015). For these reasons, the scheduling has to be more reactive and adaptable to the unforeseen change of the volatile market environment. Several studies investigated the learning and forgetting effect, but recently the scheduling problem faced more attention. The major part of works focused on the scheduling problem on a single machine (Rustogi \& Strusevich, 2014; Wang, 2007; Lee et al., 2011; Li et al. 2018a; Li et al., 2018b; Ahmadizar et al., 2013; Azadeh et al., 2017). The problem addressed is to determine the optimal sequence of the jobs to reduce the processing times. Also, the flow line is studied under the learning and forgetting effect (Bai et al., 2018; Gao et al., 2018). The simulation is used to investigate the effect of non-homogeneous learning rate on the performance of serial production stages (Ranasinghe et al., 2018). The assembly flow shop line under learning effect is studied in order to minimize the makespan, total completion time and total quadratic completion time. The scheduling problem is formulated as a mixed integer programming model. The model is solved using some metaheuristic algorithms that depend on the scale of the problem (Danyu et al., 2018). Another field of the research concerns the allocation of the workers as proposed in Liu et al. (2016). Few works were proposed investigating the flexible job shop scheduling problem with learning and forgetting considerations (Azzouz et al., 2018). This research focuses on the scheduling approach in a job shop manufacturing system introducing the learning and forgetting effect. The scheduling concerns a real-time decision on the allocation of the part to the machines with high demand volatility in terms of mix and volume. The scheduling approach proposed avoid the lot of items to react rapidly to the customer demand. The simulation environment developed allows to investigate several performance measures to test the proposed approach. The study of the flexible job shop scheduling under learning effect and deterioration is investigated using metaheuristic algorithms as a genetic algorithm and variable neighborhood search (Tayebi Araghi, 2014). These methods can be used for a limited number of jobs/parts due to the computational complexity and cannot be used to take the decision in quasi-realtime. Biel and Glock (2018) studied the effect on the key performance indicators in the multi-stage production systems of the learning and forgetting effect. In their work, they proposed flexible, real-time buffer management rules that aim at lowering excessive inventory build-up which may result from adverse input parameter combinations. The results are limited to the case studied of three multi-stages as a line. As discussed by the authors the model needs to be extended to a flexible job-shop context. The introduction of the learning effect is studied also in the case of cellular manufacturing systems as shown in Heydarian and Jolai (2018). They studied the operator allocation problem with learning effects and server breakdown in cellular manufacturing systems. The method proposed supports the decision makers in cellular manufacturing systems. A recent overview of the literature focused on learning/forgetting effects in manufacturing systems (Glock et al., 2018; Azzouz et al., 2018).

Glock et al. (2018) described the evolution of the learning curves and forgetting models and application areas. They argued as few articles considered both learning and forgetting effects and how may work on a single machine are not suitable for real industrial applications. Azzouz et al. (2018) discussed how the computational complexity of the learning/effects problems remains open. Moreover, there are a few types of research which study the impact of dynamic events such as breakdown machines, dynamic arrivals of jobs and the absence of workers on the scheduling problems under learning effects. From the analysis of the past works, the main limits of the literature are the following. Few works addressed the problem in job-shop manufacturing systems with the introduction of uncertain in demand, mix, etc. The few models proposed in this context can be introduced in manufacturing systems with determined demand and a restricted number of machines. The scheduling models proposed are characterized by relevant computational complexity with limited reaction to unforeseen events. In response, this paper seeks to propose a scheduling approach with the first research questions

RQ1: what is the performance of the scheduling model that works in real-time in a turbulent environment? 
Often, the performance measures analysed are limited to a restricted area without providing complete information about the manufacturing systems behavior.

Therefore, the second research question asks:

RQ2: what is the impact of the scheduling model on the performance of the manufacturing system under different learning-forgetting rates and demand?

The remainder of this paper is organized as follows. Section 3 describes the reference context of the study proposed. Section 3 presents the proposed scheduling approach, while Section 4 describes the simulation model, the experimental setup with the analyses and discusses the results of the simulation study. Finally, in Sect. 7, concluding remarks are made and directions for future research work are presented.

\section{Research context}

The manufacturing system is a flexible manufacturing system with complete flexibility of the machines; each machine can manufacture all the operations. The parts enter the manufacturing system follow an exponential distribution; when a part enters, an integer uniform distribution is used to assign (value 1 extracted) or not (value 0 extracted) for each possible $i$-th operation $o p_{i}$. Each part must have almost one operation to perform. Each operation $i$-th has a base process time, the learning rate allows to reduce the process time if the same parts are produced consequently; while, the forgetting rate increases the processing time when a different part is produced.

Throughout the paper, the following terminology is used:

$f_{i} \quad$ it is the exponent of the forgetting curve (forgetting rate) for stage $i$ with $0 \leq f_{i}<1$;

$l_{i} \quad$ it is the exponent of the learning curve (learning rate) for stage $i$ with $0 \leq l_{i}<1$;

$T_{1, i} \quad$ it is the time required to produce the first item on stage $i$ (the base processing time);

$W L_{i} \quad$ it is the workload of the machine $i$;

$k \quad$ it is the number of same item typology to compute the processing time by the learning function for a specific item typology;

$T_{k, j} \quad$ it is the processing time of the $j$ item typology after a number of $k$ consequently item $(j)$ manufactured following the learning function.

$T_{x, j} \quad$ it is the processing time of the $j$ item typology after a number of $\mathrm{x}$ different item form $j$ manufactured following the forgetting function.

$T_{\max }$ is the maximum value for the processing time; this value is used to limit the forgetting function.

Nop it is the number of operations assigned to the item;

$N q_{i} \quad$ it is the number of items in queue at machine $i$;

$N q_{p, i} \quad$ it is the number of items of typology $p$ in queue at machine $i$;

$\operatorname{Perc}_{p, i}$ it is the percentage of item of typology $p$ in queue of machine $\mathrm{i}$;

$\alpha, \beta$ they are the weights used in the scheduling model;

$W L c_{i} \quad$ it is the workload corrected used to allocate the item at the machine $i$;

The processing time of each machine is characterized by the learning and forgetting effects. The learning effect used in this paper is the formulation proposed by Wright (1936), the time to produce the $k$-th item on stage $i$ can be calculated as:

$$
T_{k, i}=T_{1, i} \bullet k^{-l_{i}}
$$

The forgetting occurs when other product typologies are processed; the forgetting curve used in this paper is the same proposed by Carlson and Rowe (1976):

$$
T_{x, i}=T_{1, i} \bullet x^{f_{i}}
$$


The two equations need to be combined to obtain a learning-forgetting during the manufacturing of the items for each machine. The following conditions can occur:

1) During the learning period of a machine (processing a sequence of similar items) a different product item enters the machine until a process time $T_{\text {now }}$. Then, the process time is affected by a one step of forgetting. The new process time is computed by the following expression:

$$
T_{l f, i}=\operatorname{Max}\left(T_{\text {now }}+T_{1, i} \bullet\left(x^{f_{i}}-1\right) ; T_{\max }\right)
$$

2) During the forgetting period of a machine (process time of different items), two consecutive items are manufactured. Then, the process time is affected by the learning. The new process time is computed by the following expressions. First, it is computed the $k_{e q}$ as the learning expression:

$$
k_{\text {eq }}=\left(\frac{T_{1, k}}{T_{\text {now }}}\right)^{\frac{1}{f}}
$$

Then, it is applied the learning expression (1) using the $k_{e q}$.

Fig. 1 shows an example of the processing time curve of a machine under learning and forgetting effects. From the part 1 to 10 , the typology is the same and the processing time reduces due to the learning effect; from part 11 to 13, the typology part is different and the processing time increases due to the forgetting effect. When the same part of the [1-10] arrives at the machine the processing time starts from the value due to the forgetting effect.

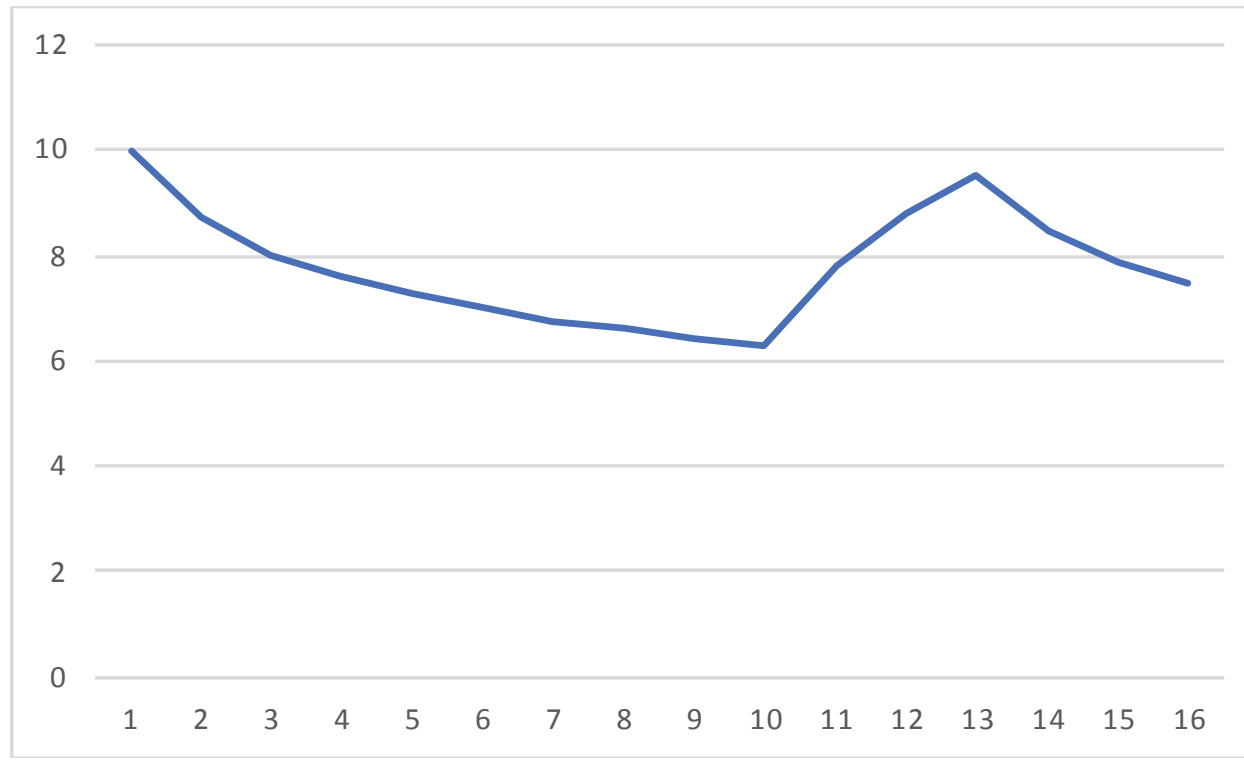

Fig. 1. Example of the learning/forgetting curve

The parts are assigned to the machines following a workload policy (benchmark model). Then, at each part is assigned a due date following the distribution:

$$
\text { duedate }=T_{\text {now }}+T_{1} \bullet N_{o p}
$$




\section{Scheduling approach}

The scheduling policy allocates the part to the machines because each machine can perform all the technological operations. The first policy is used as a benchmark to evaluate the proposed methods; this policy allocates the part to the machine with the lower workload of the parts in the queue. The workload is computed using the base processing time of the parts.

$$
W L_{i}=T_{1} \bullet N q_{i}
$$

The part is allocated to the machine $i$ with the lower value of the $W L_{i}$. This method does not take into account the potential evolution of the processing time due to the learning rate of the parts in the queue.

\subsection{Proposed approach}

The approach proposed in this paper for the scheduling problem is based on the support of a Multi-Agent Architecture. The Multi Agent Systems (MASs) is an enabling technology to support Industry 4.0 as discussed in Wang et al. (2016) and Leitão et al. (2016). The Multi-Agent Architecture consists of two types of agents: a machine agent (MA) associated to each workstation. It is an intelligent entity who manages the workstation to improve resource efficiency. Moreover, when a new part enters the system the corresponding part agent (PA) is created; it analyses the part status locating the following activities to be scheduled and performs the strategy to assign the part to the workstation. The coordination mechanism among the agents consists of the following steps (as shown in Fig. 2):

- The PA analyses the process plan of the part and determines the next technological operation to process.

- It sends a request to all MA with the information of the technological operation request; then, it waits for the answers of the MA.

- The MA computes the workload of the machine and the adjusted workload (the proposed policy) based on the technological operation required by the PA. Afterward, each MA sends to the PA the index computed.

- The PA receives the index from all MA agents and allocates the part to the MA with the best index proposed. Then, the PA communicate to the MA the assignment of the part.

- The MA update the learning and forgetting rate for each technological operation after a part completes a process.

- The PA checks if the process plan of the part is completed. If another operation is necessary the activities described are repeated.

The approach considers a workload of the machines adjusted considering the typology of the parts in the queue to improve the learning of the machine.

The correction of the workload to decide the scheduling of the parts is computed by the following steps:

- It is evaluated the workload of the machine $i$ considering the items in queue as computed in eq. (6).

- It is evaluated the percentage for each typology of the items in queue;

$$
\operatorname{perc}_{p, i}=\frac{N q_{p, i}}{N q_{i}}
$$

- It is considered the part typology $p^{*}$ to schedule and the workload is corrected with two parameters $\alpha$ ( between 0 and 1 ) and $\beta$ (greater than 1 ).

$$
W L c_{i}=\left(\alpha \cdot \operatorname{perc}_{p, i}+\left(1-\operatorname{perc}_{p, i}\right) \bullet \beta\right) \cdot\left(W L_{i}+T_{1}\right)
$$




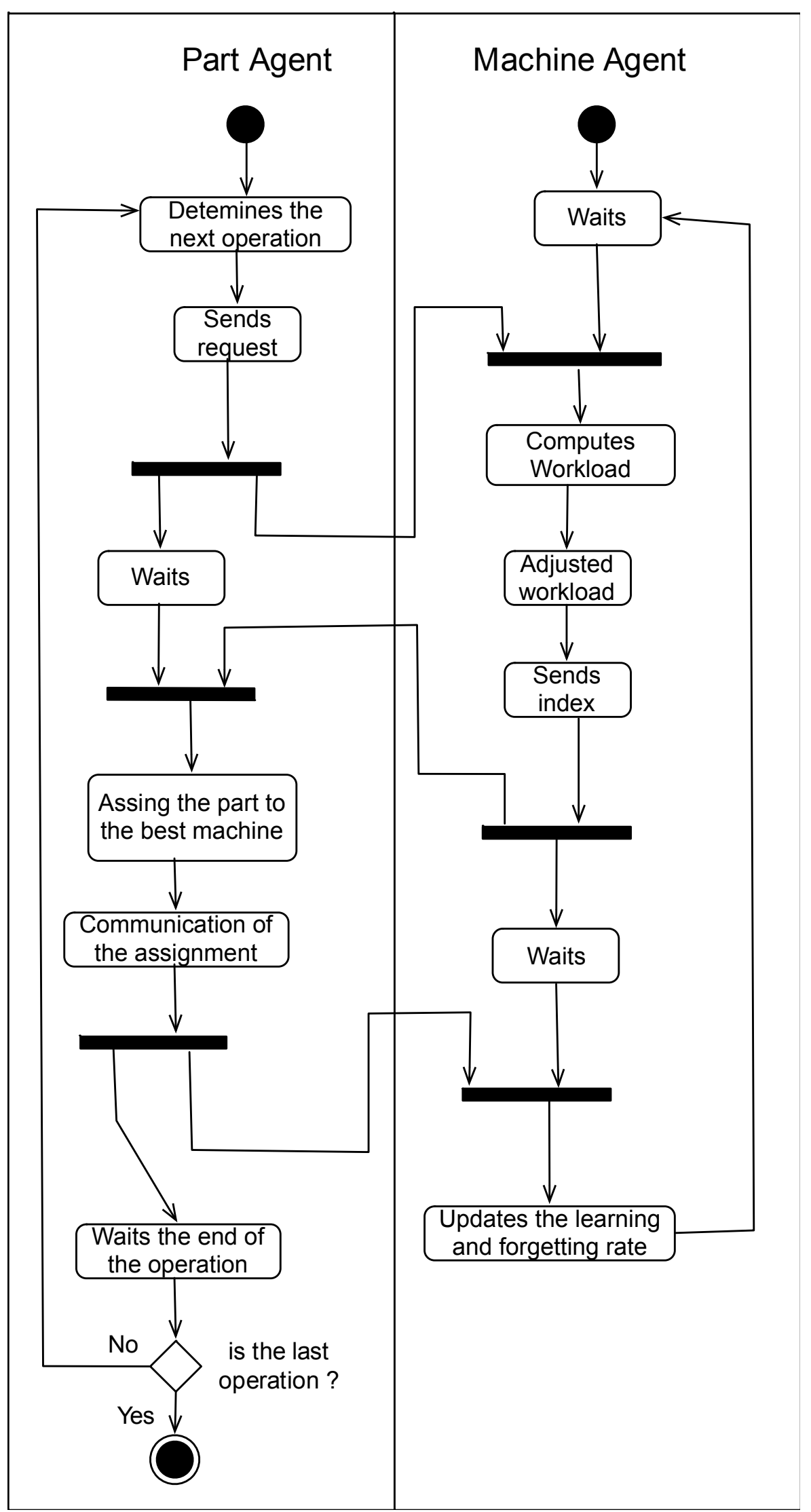

Fig. 2. UML Activity Diagram of the agents 
The workload corrected takes into account a weight to increase $\beta$ or to $\alpha$ decrease the workload of the machine. Higher is the percentage of the parts similar to the part to allocate, then the higher is the reduction by the weight $\alpha$. Otherwise, the weight $\beta$ increases the workload of the machine. The part is allocated to the machine with a lower workload corrected. In order to minimize the delay, the queues of the machines are managed by Early Due Date policy. The above model can be introduced in several manufacturing systems (job shop, cellular manufacturing etc.) and with any number of machines. The MAS allows to share the computational complexity among the agents that compose the architecture allowing decisions in quasi-real-time.

\section{Simulation Experiments and Numerical Results}

Table 1 reports the experimental factors; a full factorial design with 324 scenarios was simulated. The factors are the combination of the weight $a$ and $b$, three values for the learning and forgetting effects and three different values of the inter-arrival parameters. The inter-arrival parameters are chosen to obtain the value of the average utilisation of the manufacturing system for the benchmark about $85 \%$ (expo 8.75), $90 \%$ (expo 8.25) and 95\% (expo 7.5). Each scenario is characterized by a simulation time of 25,000 unit-time; for each scenario, a number of replications able to assure a 5\% confidence interval and $95 \%$ of confidence level for each performance measure have been conducted. Each scenario can include thousands of replications to obtain the statistical results. The due date is assigned to each part according to the following expression:

$$
\text { due date }=\text { tnow }+(\text { total processing time }) * U N I F O R M[1,3]
$$

\section{Table 1}

Experimental setting

\begin{tabular}{cc}
\hline Factors & Levels \\
\hline Scheduling & Benchmark - approach proposed \\
$\alpha-\beta$ & {$[0-2] ;[0.1-1.9] ;[0.2-1.8] ;[0.3-1.7] ;[0.4-1.6] ;[0.5-1.5]$} \\
1 & $0.1-0.2-0.3$ \\
f & $0.2-0.2-0.3$ \\
Expo & $7.5-8.25-8.75$ \\
\hline
\end{tabular}

The performance measures studied concerns the ability to deliver the part on time and the manufacturing system. The delivery parts are studied by the following performance:

- Average lateness of the parts [unit time]; it is computed the average of the delay of the parts in delay;

- Average percentage parts in delay [a-dimensional]; it is the percentage of the parts in delay using as the base the total production of the manufacturing system;

- The Standard Deviation of the lateness [unit time]; it evaluates how the variability of the delay.

The manufacturing system is evaluated by the following performance measures:

- The throughput time of the parts [unit time]; it is the total time of the parts spent in the manufacturing system;

- The average Work In Process [parts]; it is an index of the fixed assets in items.

- The average process time of the workstations [unit time]; it is the average process time of the machines during the simulation due to the learning and forgetting rates.

- The average utilisation of the workstations [a dimensional]; it is the average utilisation of all machines of the manufacturing system. 
The numerical results are reported as a percentage difference using as a base the benchmark model and the main and interaction effects are studied by Design Of Experiment (DOE). In the DOE analysis, the notations used is the following:

- A denotes the inter-arrival time with $1(8.75), 2(8.25)$ e $3(7.5)$;

- B denotes the learning factor with $1(0.1), 2(0.2)$ e $3(0.3)$;

- $\quad$ C denotes the forgetting factor with $1(0.1), 2(0.2)$ e $3(0.3)$;

Fig. 3 and Fig. 4 show the average lateness performance analysis. The proposed approach leads to the best results when the utilisation of the manufacturing system is higher (EXPO 7.5). Considering the learning and forgetting rate, the better results is obtaining when the learning rate is higher independently by the rate of the forgetting effect.

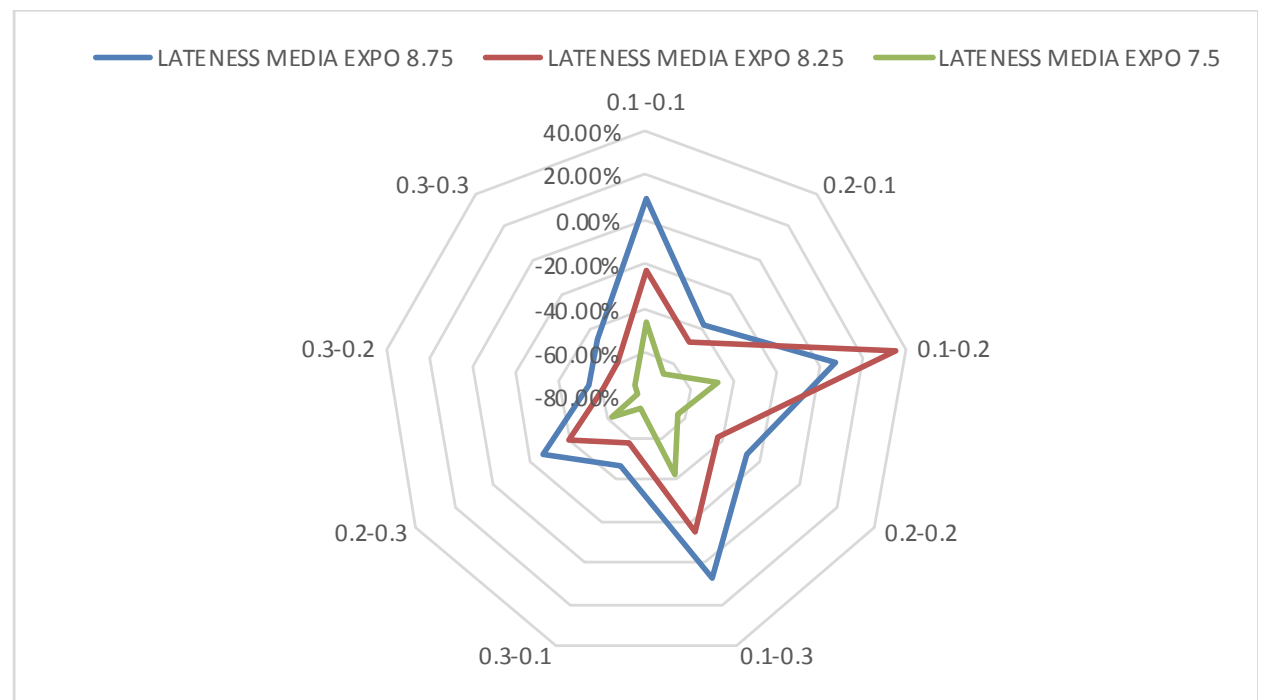

Fig. 3. Average Lateness

The main effects show how the learning rate has the more important effect on this performance, while the forgetting rate has the minor influence.
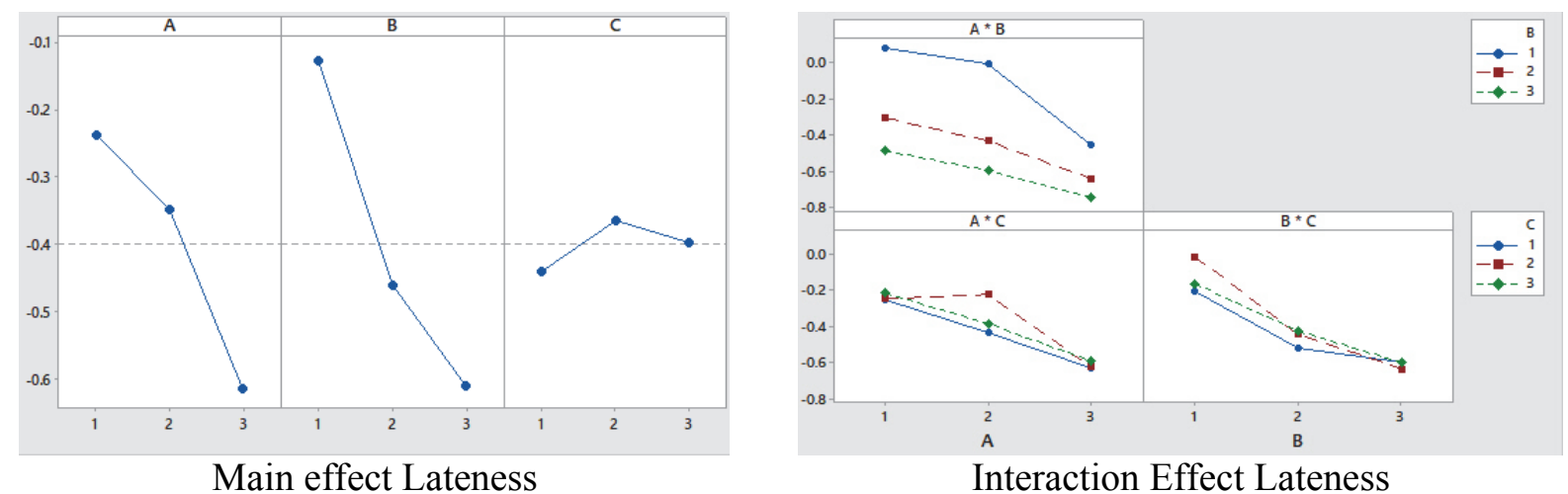

Fig. 4. Average Lateness effects

The same results are obtained for the percentage of parts in delay and standard deviations (figures 5 and 6). For this performance, the above consideration is more relevant among the factors considered. 


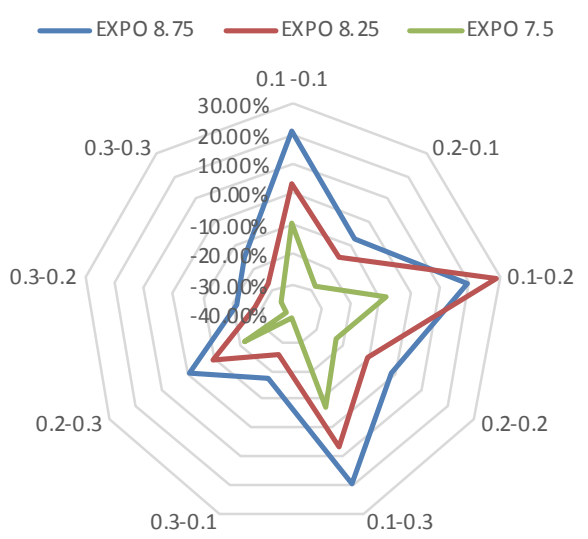

Fig. 5. Average Percentage of parts delay
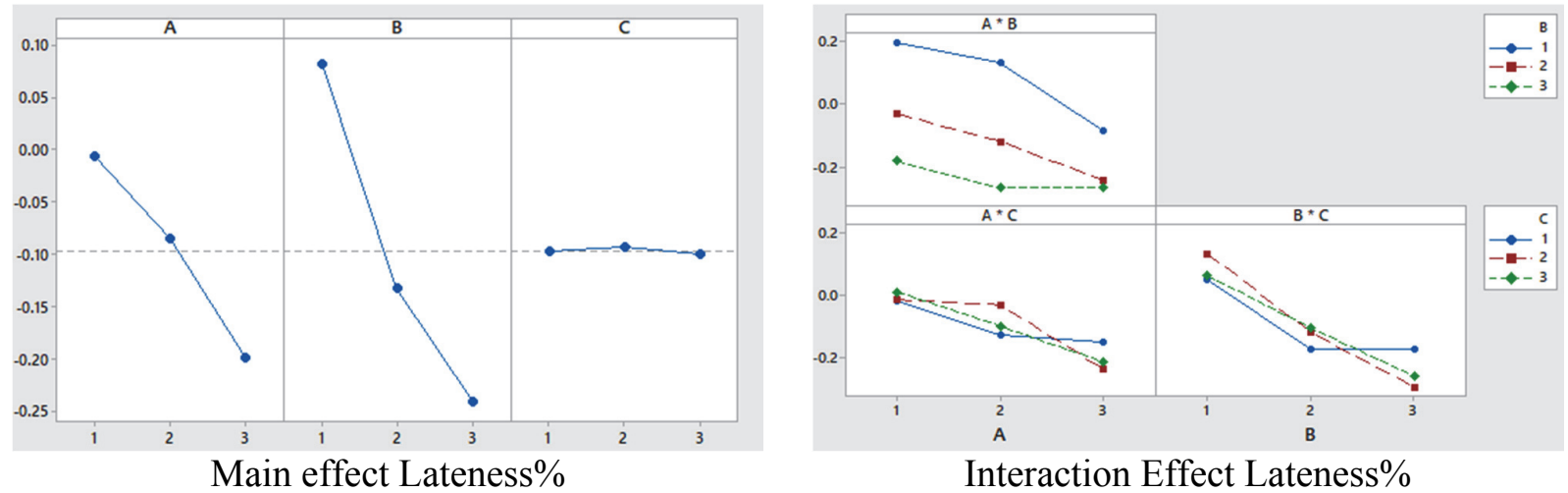

Fig. 6. Effects analysis Average Percentage of parts delay

The manufacturing performance measures have some difference from the above results. Fig. 7 and Fig. 8 reports the throughput time analysis. In this case, the better results are obtained in the case of EXPO 7.5 , but the difference between the other two values studied is very low compared to the delay performance. The main effects are due to the inter-arrival parameter, while the effect of the learning rate is relevant form the first value to the second and lower with the more increment to the third value of the rate.

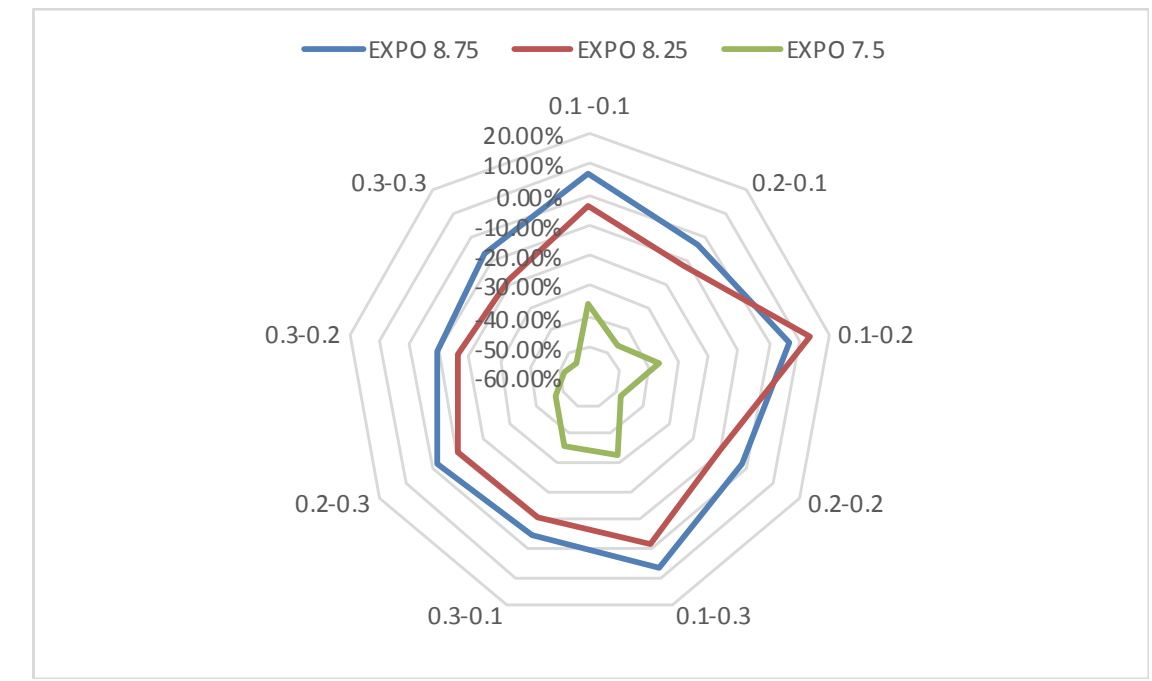

Fig. 7. Average Throughput time 


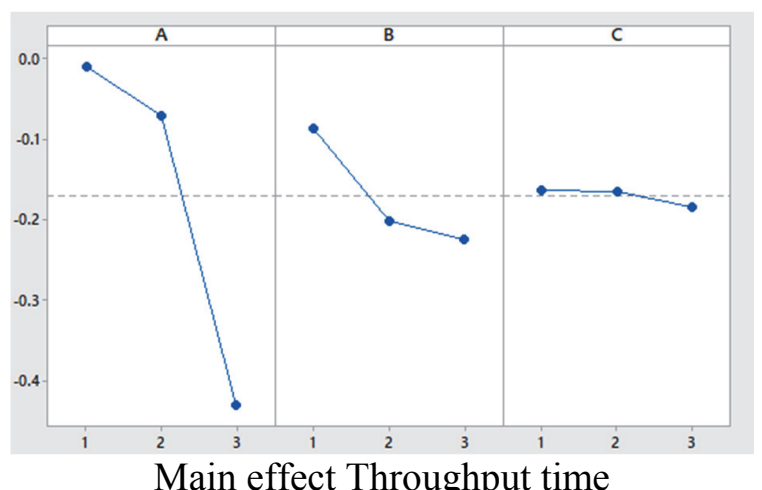

Main effect Throughput time

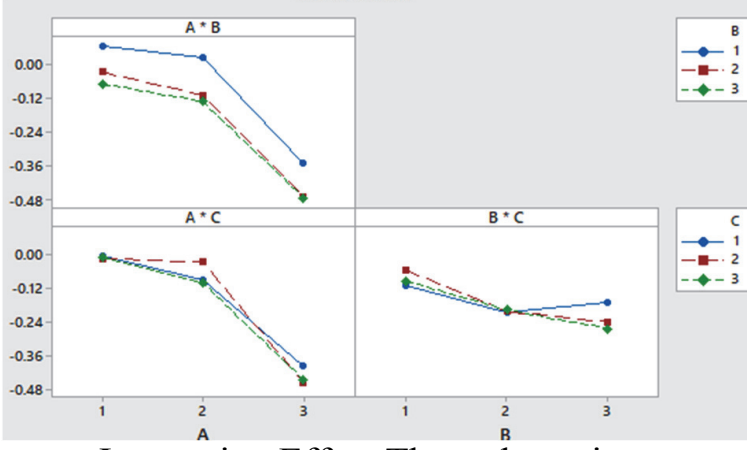

Interaction Effect Throughput time

Fig. 8. Effect Analysis Average Throughput time

Fig. 9 and Fig. 10 report the analysis of the work in process. The main difference compare to the above comments are the following. In this case, the learning rate has a positive effect from the rate 0.1 to 0.2 , while the effect is negative is the rate grows up to 0.3. Moreover, the effect of forgetting rate is relevant to increase the work in process of the manufacturing system.

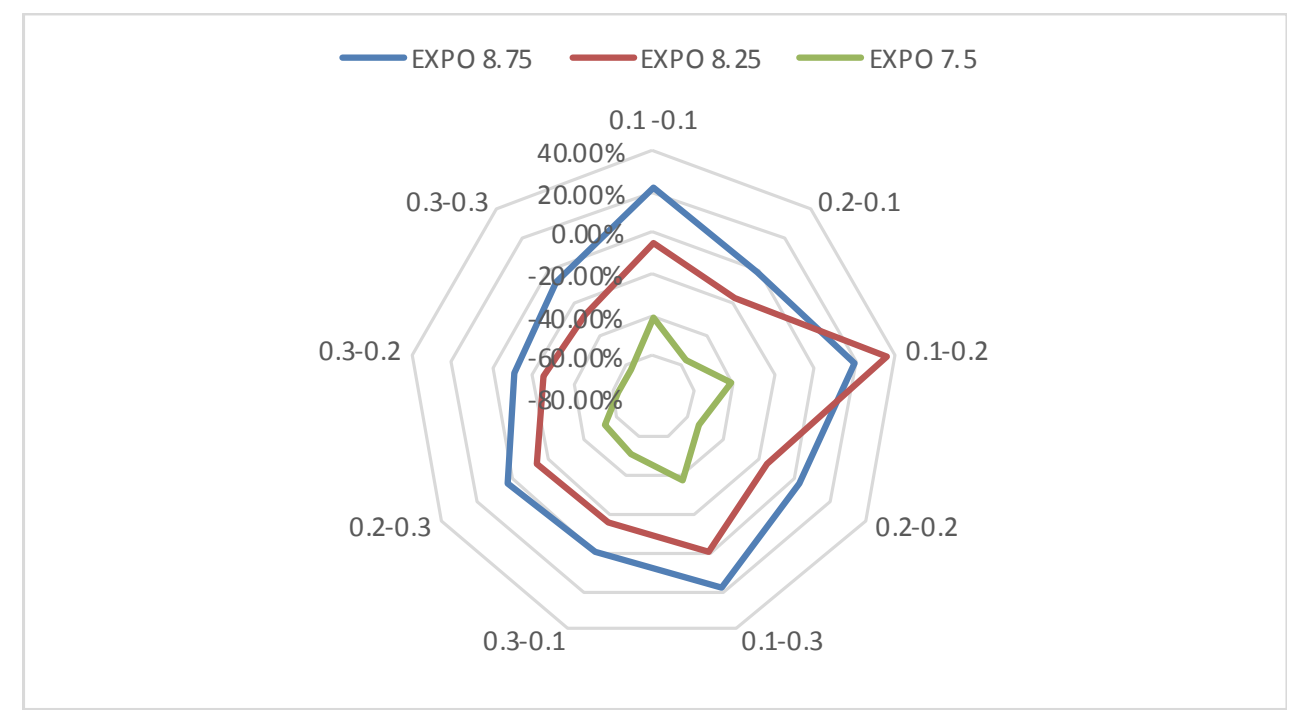

Fig. 9. Work In Process
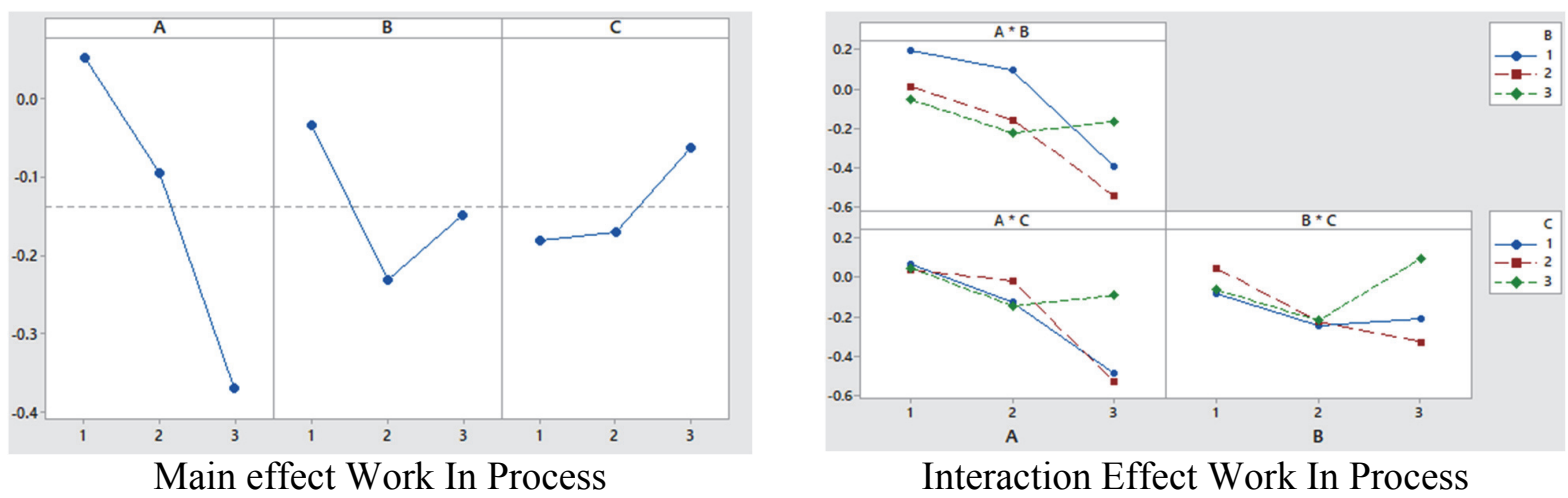

Interaction Effect Work In Process

Fig. 10. Work In Process 
The results of the average process time are given in Fig. 11 and Fig. 12. The inter-arrival time does not affect the difference of the process time from the benchmark model. The main factor is obviously the learning rate on the processing time. However, the forgetting rate has a lower influence on this performance. The average reduction of the process time due to the proposed approach is limited compared to the benchmark model.

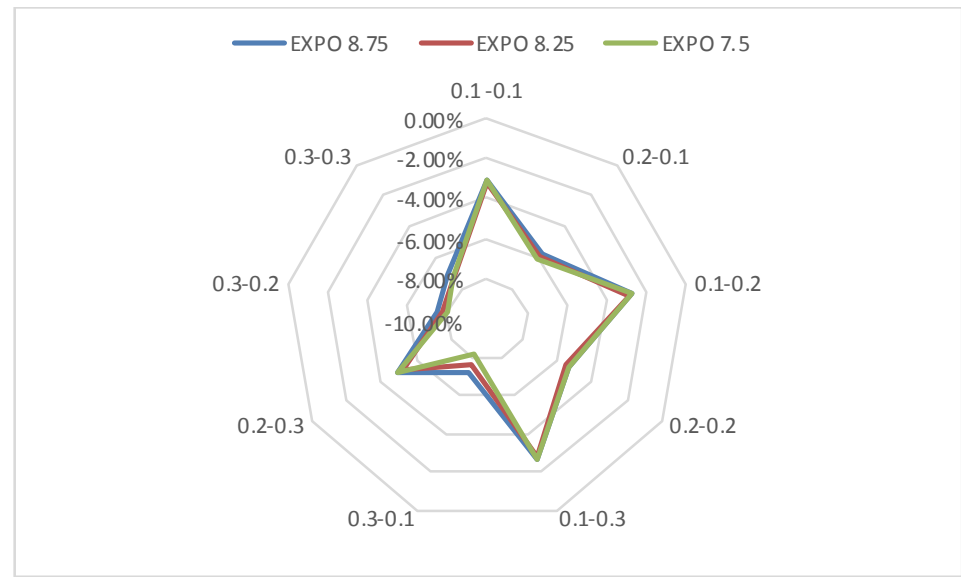

Fig. 11. Average Process Time
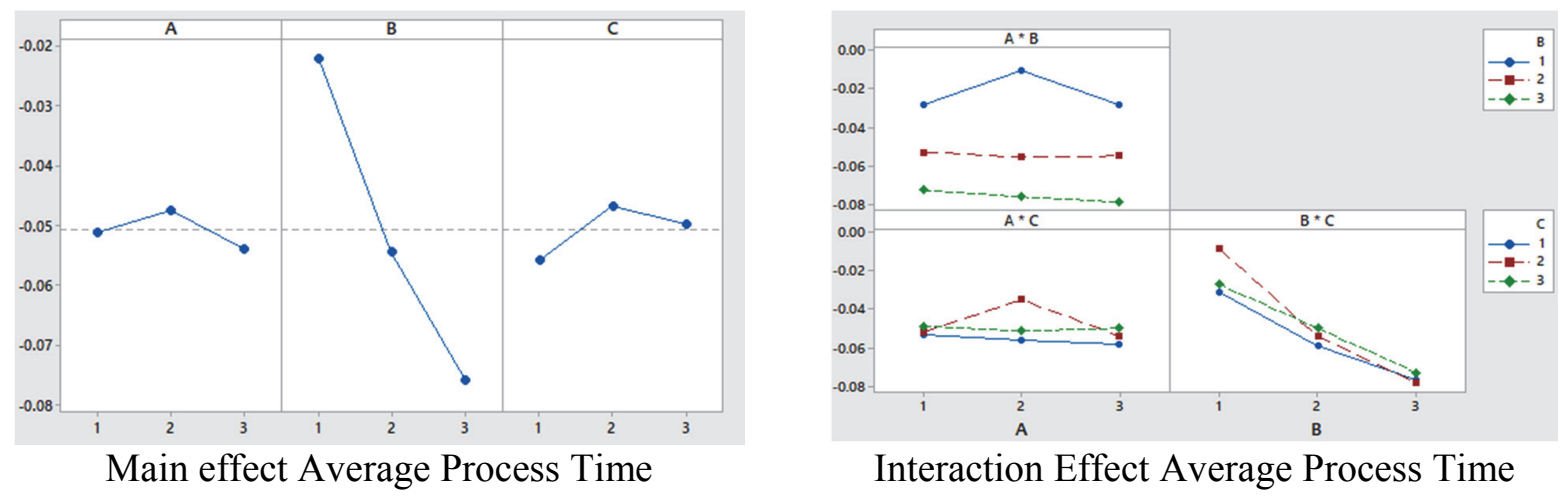

Fig. 12. Effect analysis - Average Process Time

Fig. 13 and Fig. 14 show the average utilisation of the manufacturing system. The proposed approach allows to reduce the utilisation of the manufacturing system with the same throughput level. The better results are obtained for the higher or lower inter-arrival time.

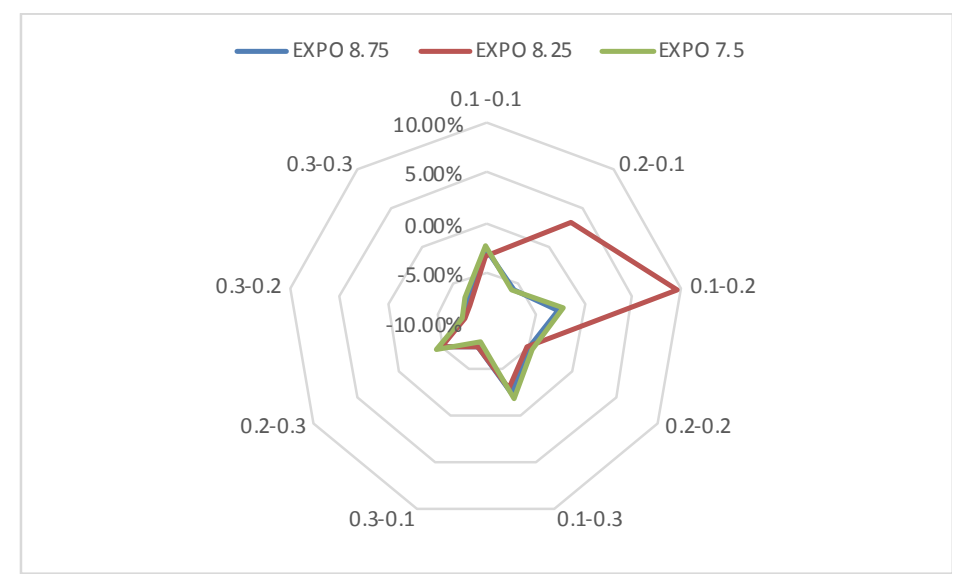

Fig. 13. Average Utilisation 

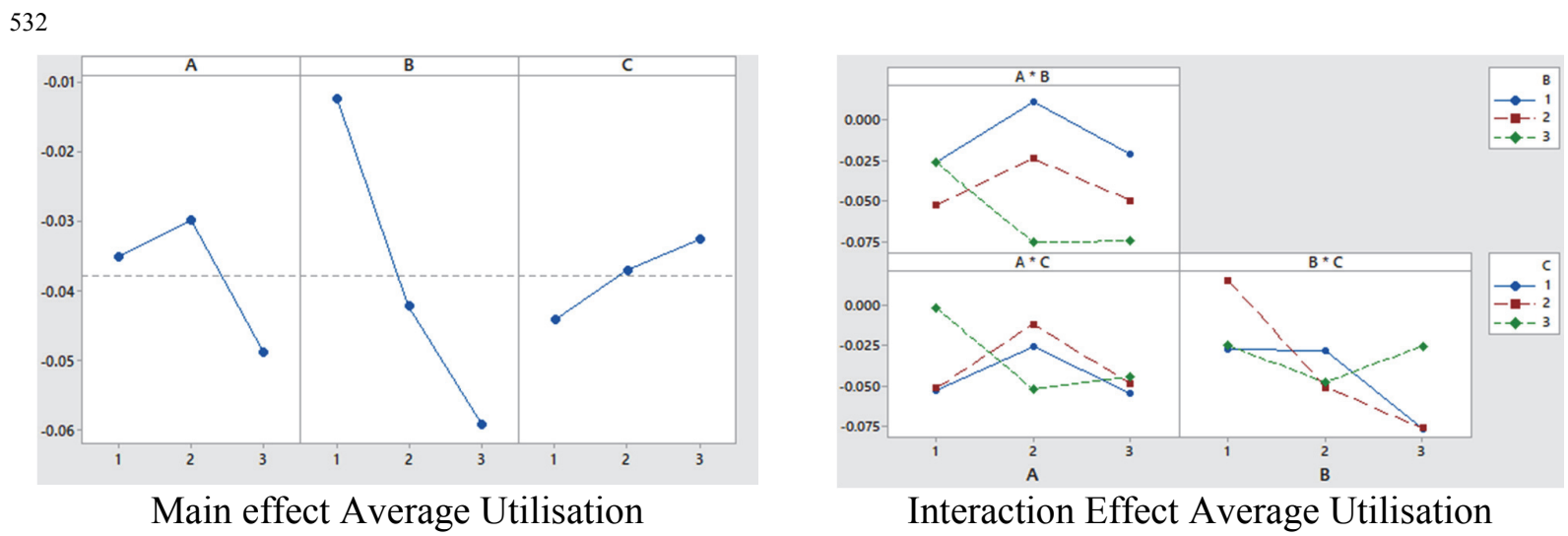

Fig. 14. Effect analysis - Average utilisation

From the above results the following issue can be reassumed:

- The proposed approach allows improving all the performance studied. The improvements are more relevant when the inter-arrival of the parts is lower. The scheduling is more relevant when the number of items in queue is higher.

- The greater improvements are obtained on the reduction of lateness, the percentage of the parts in delay and the standard deviation of the lateness. Then, the proposed approach works mainly on the delivery performance.

- The learning rata has the main influence on the performance, while the forgetting rate has a lower impact. This is relevant because is more important to improve the learning than the forgetting rate.

\section{Conclusions and future development paths}

Although a broad literature on learning effect on manufacturing system exists, specific works on learning and forgetting together are scarce and are focused on a single machine or simplified configuration of machines as flow shop. In response, the first research question asked: "what is the performance of the scheduling model that works in real-time in a turbulent environment?" The simulation environment developed to support the analysis of the proposed scheduling approach compared to the benchmark model. The results show how the proposed approach improves the performance in different conditions of demand (inter-arrival time of the parts) with a more complex manufacturing system. The scheduling approach can be extended to different configurations of the manufacturing system by the use of the multi agent architecture. The second research question asked: "what is the impact of the scheduling model on the performance of the manufacturing system under different learning-forgetting rates and demand?"

Using the simulation, we demonstrated that the performance measures improve under different values of learning and forgetting rates. Moreover, the learning effect has more influence on the results than the value of the forgetting rates. This drive the efforts to the learning than the forgetting issue. The managerial implications concern the support for the decision maker for the scheduling of a manufacturing system under learning and forgetting effect. The map of the performance obtained helps the decision maker on the benefits derived by the investment in the learning rate. Multi-agent architecture can be integrated into the modern manufacturing system under industry 4.0 development. This work drives the decision maker to invest to improve the learning rate that is the main factor for the performance. Moreover, the use of the simulation allows highlighting what is the improvement in the different conditions of the manufacturing system considering a wider range of performance measures. The limitation of this study is that we assume the same learning and forgetting functions for all the machines and parts. The learning and forgetting function can depend on the machine or the particular part processed. Furthermore, it may be investigated the introduction of the workload control approach that allows forming lots of part to schedule in the manufacturing system. 


\section{References}

Ahmadizar, F., \& Hosseini, L. (2013). Minimizing Makespan in a Single-machine Scheduling Problem with a Learning Effect and Fuzzy Processing times. The International Journal of Advanced Manufacturing Technology, 65(1-4), 581-587.

Azadeh, A., Habibnejad-Ledari, H., Abdolhossein Zadeh, S., and Hosseinabadi Farahani, M. (2017). A Single-machine Scheduling Problem with Learning Effect, Deterioration and Non-monotonic Timedependent Processing times. International Journal of Computer Integrated Manufacturing, 30(2-3): 292-304.

Azzouz, A., Ennigrou, M., \& Ben Said, L. (2018). Scheduling problems under learning effects: classification and cartography. International Journal of Production Research, 56(4), 1642-1661.

Bai, D., Tang, M., Zhang, Z. H., \& Santibanez-Gonzalez, E. D. (2018). Flow shop learning effect scheduling problem with release dates. Omega, 78, 21-38.

Biel, K., \& Glock, C.H. (2018). Governing the dynamics of multi- stage production systems subject to learning and forgetting effects: A simulation study. International Journal of Production Research, 56(10), 3439-3461

Biskup, D. (2008). A state-of-the-art review on scheduling with learning effects. European Journal of Operational Research, 188(2), 315-329.

Carlson, J. G., \& Rowe, A. J. (1976). How much does forgetting cost. Industrial Engineering, 8(9), 4047.

Gao, F., Liu, M., Wang, J. J., \& Lu, Y. Y. (2018). No-wait two-machine permutation flow shop scheduling problem with learning effect, common due date and controllable job processing times. International Journal of Production Research, 56(6), 2361-2369.

Outlook, G. M. (2015). Preparing for battle: Manufacturers get ready for transformation. KPMG.2015.-34 p.[Web resource].—link: https://www. kpmg. com/CN/en/IssuesAndInsights/ArticlesPublications/Documents/Global-Manufacturing-Outlook-O201506. $p d f$.

Globerson, S., Levin, N., \& Shtub, A. (1989). The impact of breaks on forgetting when performing a repetitive task. IIE transactions, 21(4), 376-381.

Glock, C. H., Grosse, E. H., Jaber, M. Y., \& Smunt, T. L. (2018). Applications of learning curves in production and operations management: A systematic literature review. Computers \& Industrial Engineering. https://doi.org/10.1016/j.cie.2018.10.030.

Heydarian, D., \& Jolai, F. (2018). Simulation optimization of operator allocation problem with learning effects and server breakdown under uncertainty. Production \& Manufacturing Research, 6(1), 396415.

Lee, W-C , Wu, C-C., \& Hsu, P-H. (2011). A single-machine learning effect scheduling problem with release times. Omega, 38(1), 3-11.

Leitão, P., Colombo, A. W., \& Karnouskos, S. (2016). Industrial automation based on cyber-physical systems technologies: Prototype implementations and challenges. Computers in Industry, 81, 11-25.

Li, L., Yan, P., Ji, P., \& Wang, J. B. (2018a). Scheduling jobs with simultaneous considerations of controllable processing times and learning effect. Neural computing and applications, 29(11), 1155 1162.

Li, X., Jiang, Y., \& Ruiz, R. (2018). Methods for scheduling problems considering experience, learning, and forgetting effects. IEEE Transactions on Systems, Man, and Cybernetics: Systems, 48(5), 743754.

Liu, C., Wang, J., \& Leung, J. Y. T. (2016). Worker assignment and production planning with learning and forgetting in manufacturing cells by hybrid bacteria foraging algorithm. Computers \& Industrial Engineering, 96, 162-179.

Nembhard, D. A., \& Shafer, S. M. (2008). The effects of workforce heterogeneity on productivity in an experiential learning environment. International journal of production research, 46(14), 3909-3929. 
Ranasinghe, T., Senanayake, C. D., \& Perera, K. (2018, May). Effects of Non-Homogeneous Learning on the Performance of Serial Production Systems-A Simulation Study. In 2018 Moratuwa Engineering Research Conference (MERCon) (pp. 162-166). IEEE.

Rustogi, K., \& Strusevich, V. A. (2014). Combining time and position dependent effects on a single machine subject to rate-modifying activities. Omega, 42(1), 166-178.

Shafer, S. M., Nembhard, D. A., \& Uzumeri, M. V. (2001). The effects of worker learning, forgetting, and heterogeneity on assembly line productivity. Management Science, 47(12), 1639-1653.

Tayebi Araghi, M. E., Jolai, F., \& Rabiee, M. (2014). Incorporating learning effect and deterioration for solving a SDST flexible job-shop scheduling problem with a hybrid meta-heuristic approach. International Journal of Computer Integrated Manufacturing, 27(8), 733-746.

Wang, S., Wan, J., Zhang, D., Li, D., \& Zhang, C. (2016). Towards smart factory for industry 4.0: a selforganized multi-agent system with big data based feedback and coordination. Computer Networks, 101, 158-168.

Wang, J. B. (2007). Single-machine scheduling problems with the effects of learning and deterioration. Omega, 35(4), 397-402.

Wright, T. P. (1936). Factors Affecting the Cost of Airplanes. Journal of the Aeronautical Sciences, 3(4), $122-128$.

Yelle, L. E. (1979). The Learning Curve: Historical Review and Comprehensive Survey. Decision Sciences, 10(2), 302-328.

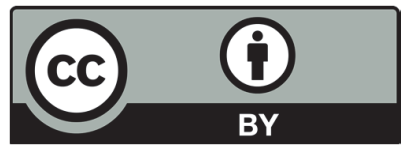

(C) 2019 by the authors; licensee Growing Science, Canada. This is an open access article distributed under the terms and conditions of the Creative Commons Attribution (CCBY) license (http://creativecommons.org/licenses/by/4.0/). 